\title{
Correction to: Relative Age Effects Across and Within Female Sport Contexts: A Systematic Review and Meta-Analysis
}

\author{
Kristy L. Smith ${ }^{1} \cdot$ Patricia L. Weir ${ }^{1} \cdot$ Kevin Till $^{2} \cdot$ Michael Romann ${ }^{3}$. \\ Stephen Cobley $^{4}$ (D)
}

Published online: 17 April 2018

(C) Springer International Publishing AG, part of Springer Nature 2018

\section{Correction to: Sports Med \\ https://doi.org/10.1007/s40279-018-0890-8}

An Online First version of this article was made available at https://link.springer.com/article/10.1007/s40279-0180890-8 on 13 March 2018. Some errors were subsequently identified by the authors, particularly in regard to Table 4. Although the details pertained to in the table were correct in the original manuscript, it appears that errors were introduced during production of the article. The published article has now been updated with a corrected version of Table 4 . This corrected version of the table is also shown below.

The original article can be found online at https://doi.org/10.1007/ s40279-018-0890-8.

Stephen Cobley

stephen.cobley@sydney.edu.au

1 Faculty of Human Kinetics, University of Windsor, Windsor, ON, Canada

2 Institute for Sport, Physical Activity and Leisure, Leeds Beckett University, Leeds, UK

3 Swiss Federal Institute of Sport Magglingen, Magglingen, Switzerland

4 Exercise and Sport Sciences, Faculty of Health Sciences, The University of Sydney, Cumberland Campus, 75 East St,

Lidcombe, Sydney, NSW 2141, Australia 
Table 4 Summary of quartile $(Q 1)$ vs. quartile $(Q 4)$ subgroup analyses according to identified moderating factors

\begin{tabular}{|c|c|c|c|c|c|c|c|c|c|c|}
\hline \multicolumn{2}{|l|}{ Random-effects model } & \multicolumn{3}{|c|}{ Subgroup estimates } & \multicolumn{3}{|c|}{$\begin{array}{l}\text { Mixed-effects between subgroup } \\
\text { analysis }\end{array}$} & \multicolumn{3}{|c|}{ Subgroup heterogeneity } \\
\hline $\begin{array}{l}\text { Moderator variable } \\
\text { Subgroup }\end{array}$ & $\begin{array}{l}\text { (No. of } \\
\text { samples) }\end{array}$ & $\begin{array}{l}\text { Point } \\
\text { estimate }^{a}\end{array}$ & $95 \% \mathrm{CI}$ & $Z$ value $^{\mathrm{b}}$ & $p$ value $^{\mathrm{c}}$ & $\begin{array}{l}Q^{\mathrm{d}} \text { Between } \\
\text { value }\end{array}$ & $p$ value & $\begin{array}{l}Q \text { in } \\
\text { subgroup } \\
Q \text { within }\end{array}$ & $\begin{array}{l}p \text { in } \\
\text { subgroup } \\
p \text { within }\end{array}$ & $\begin{array}{l}I^{2} \\
\text { subgroup }^{\mathrm{e}}\end{array}$ \\
\hline \multicolumn{11}{|l|}{ Age } \\
\hline $\begin{array}{l}\text { Pre-adolescent } \\
{[\leq 11 \mathrm{y}]}\end{array}$ & (51) & 1.33 & $1.25-1.42$ & 8.68 & 0.0001 & & & 238.13 & 0.0001 & 79.00 \\
\hline $\begin{array}{l}\text { Adolescent }[12-14 \\
\text { y] }\end{array}$ & (55) & 1.28 & $1.19-1.37$ & 7.05 & 0.0001 & & & 241.83 & 0.0001 & 77.67 \\
\hline $\begin{array}{l}\text { Post-adolescent } \\
{[15-19 \mathrm{y}]}\end{array}$ & (91) & 1.14 & $1.08-1.20$ & 4.79 & 0.0001 & & & 707.57 & 0.0001 & 87.28 \\
\hline Adult $[>19 \mathrm{y}]$ & $(32)$ & 1.08 & $0.97-1.19$ & 1.44 & 0.14 & & & 55.10 & 0.005 & 43.74 \\
\hline \multirow{2}{*}{$\begin{array}{l}\text { Not codable into } \\
\text { above }\end{array}$} & (79) & 1.37 & $1.29-1.46$ & 9.74 & 0.0001 & 31.24 & 0.0001 & 369.12 & 0.0001 & 78.86 \\
\hline & & & & & & & & 1611.78 & 0.0001 & \\
\hline \multicolumn{11}{|l|}{ Competition level } \\
\hline Recreational & (76) & 1.08 & $1.02-1.14$ & 2.83 & 0.005 & & & 1028.85 & 0.0001 & 92.71 \\
\hline Competitive & (71) & 1.39 & $1.30-1.50$ & 9.38 & 0.0001 & & & 243.92 & 0.0001 & 71.30 \\
\hline Representative & (44) & 1.45 & $1.31-1.61$ & 7.24 & 0.0001 & & & 126.83 & 0.0001 & 66.09 \\
\hline Elite adolescent & (5) & 2.70 & $1.76-4.12$ & 4.58 & 0.0001 & & & 6.64 & 0.15 & 39.81 \\
\hline $\begin{array}{l}\text { Elite post- } \\
\text { adolescent }\end{array}$ & (18) & 1.65 & $1.41-1.92$ & 6.48 & 0.0001 & & & 35.92 & 0.005 & 52.67 \\
\hline Elite adult & (12) & 1.27 & $1.02-1.50$ & 2.19 & 0.02 & & & 9.20 & 0.60 & 0.00 \\
\hline $\begin{array}{l}\text { Elite, combination } \\
\text { of age }\end{array}$ & (26) & 1.42 & $1.26-1.61$ & 5.65 & 0.0001 & & & 56.16 & 0.0001 & 55.48 \\
\hline \multirow{2}{*}{$\begin{array}{l}\text { Not codable into } \\
\text { above }\end{array}$} & (56) & 1.19 & $1.12-1.27$ & 5.40 & 0.0001 & 77.09 & 0.0001 & 357.62 & 0.0001 & 84.62 \\
\hline & & & & & & & & 1865.17 & 0.0001 & \\
\hline \multicolumn{11}{|l|}{ Sport type } \\
\hline Team & (154) & 1.33 & $1.27-1.39$ & 12.51 & 0.0001 & & & 689.01 & 0.0001 & 77.79 \\
\hline Individual & (154) & 1.18 & $1.12-1.24$ & 5.26 & 0.0001 & & & & & \\
\hline $\begin{array}{l}\text { Physically } \\
\text { demanding }\end{array}$ & (88) & 1.23 & $1.16-1.30$ & 7.19 & 0.0001 & & & 1125.83 & 0.0001 & 92.82 \\
\hline $\begin{array}{l}\text { Technique (skill) } \\
\text { based }\end{array}$ & (59) & 1.06 & $0.97-1.16$ & 1.36 & 0.17 & & & 118.20 & 0.0001 & 51.77 \\
\hline \multirow[t]{2}{*}{$\begin{array}{l}\text { Weight } \\
\text { categorised }\end{array}$} & (7) & 1.18 & $0.93-1.51$ & 1.38 & 0.16 & 20.58 & 0.001 & 7.48 & 0.27 & 19.81 \\
\hline & & & & & & & & 2040.54 & 0.0001 & \\
\hline \multicolumn{11}{|l|}{ Study Quality } \\
\hline $\begin{array}{l}\text { Lower (scores } \\
5-9 \text { ) }\end{array}$ & (38) & 1.63 & $1.46-1.82$ & 8.55 & 0.0001 & & & 72.48 & 0.0001 & 48.95 \\
\hline Medium [10-11] & (92) & 1.29 & $1.22-1.37$ & 8.72 & 0.0001 & & & 348.55 & 0.0001 & 73.89 \\
\hline \multirow[t]{2}{*}{ Higher [12-14] } & (178) & 1.19 & $1.14-1.25$ & 8.46 & 0.0001 & 27.44 & 0.001 & 1596.47 & 0.0001 & 88.91 \\
\hline & & & & & & & & 2017.51 & 0.0001 & \\
\hline
\end{tabular}

CI confidence interval

${ }^{\text {a }}$ Point estimate $=$ pooled overall odds ratio $(Q 1$ vs. $Q 4)$ estimate

${ }^{\mathrm{b}} Z$ value $=$ reflects the test for an overall effect

${ }^{c} p=$ indicating probability of significance $(p \leq 0.05)$

${ }^{\mathrm{d}} Q$ value $=$ dispersion of studies about the point estimate overall or within the subgroup

${ }^{\mathrm{e}} I^{2}=$ reflects heterogeneity within the subgroup 